\title{
Safety of Tamoxifen During Pregnancy: 3 Case Reports and Review of the Literature
}

\author{
Emre Koca ${ }^{a}$ Taha Y. Kuzan ${ }^{a}$ Taner Babacan ${ }^{a}$ Ibrahim H. Turkbeyler ${ }^{b}$ \\ Furkan Sarıcı $^{a}$ Kadri Altundag ${ }^{a}$ \\ ${ }^{a}$ Hacettepe University Cancer Institute, Ankara; ${ }^{b}$ Department of Internal Medicine, Adıyaman University School of Medicine, Turkey
}

\section{Keywords \\ Breast cancer · Pregnancy · Tamoxifen}

\section{Schlüsselwörter}

Mammakarzinom · Schwangerschaft · Tamoxifen

Dear Editors,

Tamoxifen is one of the treatment options for estrogen receptor (ER)-positive breast cancers, and it is considered to be a teratogenic agent. Animal models have shown that tamoxifen can cause genitourinary developmental defects [1]. Although there is 1 case report showing that tamoxifen can cause genital defects in humans [2], other case studies report delivery of healthy babies by women using tamoxifen $[3,4]$. Here we report on 3 breast cancer patients who became pregnant while undergoing tamoxifen therapy.

The first case, a 32-year-old woman, presented with a left breast lump in 2007; incisional biopsy revealed infiltrative ductal carcinoma. The disease was staged as cT4cN2M1. The patient had bone metastasis at the time of diagnosis. Immunohistochemical analysis showed this tumor to be ER-positive $(100 \%(+))$, progesterone receptor (PR) $100 \%(+)$, and HER2-negative. The patient received adjuvant tamoxifen following adjuvant chemotherapy including paclitaxel, carboplatin and doxorubicin and radiotherapy. The patient became pregnant in 2009 while on a 6-month tamoxifen therapy. She discontinued tamoxifen when she discovered she was pregnant, so tamoxifen therapy lasted only 1.5 months. The patient delivered a healthy baby in 2010. After birth, positron emission tomography-computed tomography revealed regression of bone metastases. The second case, a 23-year-old woman, underwent right modified radical mastectomy for infiltrating ductal carcinoma in 2009. The disease was staged as T2N0M0. Immunohistochemical analysis showed this tumor to be ER 5\% (+), PR 50\% (+), and HER2-positive $(3+)$. The patient received adjuvant chemotherapy including paclitaxel, carboplatin, doxorubicin and trastuzumab. Adjuvant tamoxifen and leuprolide were also recommended. No radiotherapy was administered. The patient received tamoxifen for 26 months and discontinued treatment of her own accord. 1 month after that she became pregnant and took no further therapy. She delivered a healthy baby in October 2012, and is herself in good health at present. The third case, a 35-year-old woman, underwent breast-conserving surgery and sentinel lymph node dissection for infiltrating ductal carcinoma in 2011. The disease was staged as T1N0M0. Immunohistochemical analysis showed this tumor to be ER 90\% (+), PR 10\% (+), and HER2-positive (2+), confirmed by fluorescent in situ hybridization. She did not receive any adjuvant chemotherapy except tamoxifen following radiotherapy. The patient became pregnant while taking tamoxifen for 6 months in 2011. She discontinued tamoxifen when 4 weeks pregnant and had a voluntary medical abortion at 6 weeks gestation. She subsequently continued tamoxifen treatment.

Tamoxifen is a non-steroidal anti-estrogen agent used in the treatment of ER-positive breast cancers. It is contraindicated during pregnancy due to teratogenic effects, and recommended non-hormonal contraceptive methods may cause patients to get pregnant while on tamoxifen therapy. Von Schoultz et al. [5] have suggested that women taking tamoxifen who wish to become pregnant should stop tamoxifen at least 2 months prior to conception because of the drug's long half-life. Although there is insufficient knowledge regarding the use of tamoxifen and pregnancy outcomes in humans, there are some case reports indicating healthy deliveries [3, 4]. In contrast, there is 1 case report of a baby with ambiguous genitalia suggestive of a tamoxifen effect on the fetus [2]. Another case report showed a baby with congenital cranio-facial defects, who was exposed to cocaine, marijuana, and a bone scan along with tamoxifen during fetal development.

The effects of tamoxifen during pregnancy were described in the study by Braems et al. [6], based on records provided by AstraZeneca. The AstraZeneca Safety Database registered 11 babies with congenital malformations out of 44 live births, which is 1 infant with malformations for every 4 live births. In fact, the actual number of malformations was even higher, because there were 6 terminations and 2 stillbirths with fetal

\section{KARGER}

Fax +497614520714

Information@Karger.com

www.karger.com (c) 2013 S. Karger GmbH, Freiburg

$1661-3791 / 13 / 0086-0453 \$ 38.00 / 0$

Accessible online at:

www.karger.com/brc
Taner Babacan, MD

Department of Medical Oncology

Hacettepe University Institute of Oncology

Sihhiye Ankara 06100, Turkey

mdbabacan@mynet.com 
defects, compared with 17 terminations and 1 stillbirth without fetal defects, which is a total rate of nearly 1 in 3 births [6].

We herein report 3 breast cancer cases in which the patients became pregnant while taking tamoxifen therapy. In the first case, the patient had a healthy baby who is now 2 years old and has no abnormalities. The second patient had an uncomplicated pregnancy and a normal healthy baby. The patient in the last case had an elective abortion after a discussion with her physician regarding the possible side effects of the chemotherapy. To conclude, due to the lack of long-term data on outcome and studies showing teratogenicity, use of tamoxifen during pregnancy is contraindicated despite reports of healthy babies after tamoxifen exposure. Premenopausal breast cancer patients who are taking tamoxifen should practice meticulous use of contraception, and they must be informed about the possible teratogenic effects of tamoxifen as well as the lack of long-term data on this subject.

\section{References}

1 Iguchi T, Hirokawa M, Takasugi M: Occurrence of genital tract abnormalities and bladder hernia in female mice exposed neonatally to tamoxifen. Toxicology 1986;42:1-11.

2 Tewari K, Bonebrake RG, Asrat T, Shanberg AM: Ambiguous genitalia in infant exposed to tamoxifen in utero. Lancet 1997;350:183.
3 Oksuzoglu B, Guler N: An infertile patient with breast cancer who delivered a healthy child under adjuvant tamoxifen therapy. Eur J Obstet Gynecol Reprod Biol 2002;104:79.

4 Isaacs RJ, Hunter W, Clark K: Tamoxifen as systemic treatment of advanced breast cancer during pregnancy-case report and literature review. Gynecol Oncol 2001;80:405-8.
5 Von Schoultz E, Johansson H, Wilking N, Rutqvist LE: Influence of prior and subsequent pregnancy on breast cancer prognosis. J Clin Oncol 1995;13:430-4.

6 Braems G, Denys H, De Wever O, Cocquyt V, Van den Broecke R: Use of tamoxifen before and during pregnancy. Onkologist 2011;16:1547-51. 